INPLASY

PROTOCOL

To cite: Niu et al. Prognostic Significance of Pretreatment Controlling Nutritional Status Score in urological cancers: A systematic review and metaanalysis. Inplasy protocol 2020120111. doi:

10.37766/inplasy2020.12.0111

Received: 22 December 2020

Published: 23 December 2020

Corresponding author: Juan Bao

bj901120@yeah.net

Author Affiliation:

Shanghai Public Health

Clinical Center of Fudan

University

Support: KY-GW-2018-46.

Review Stage at time of this submission: Data analysis.

Conflicts of interest:

None.

\section{Prognostic Significance of Pretreatment Controlling Nutritional Status Score in urological cancers: A systematic review and meta-analysis}

Niu, X1; Zhu, Z2; Bao, J3.

Review question / Objective: Controlling Nutritional Status (CONUT) score is a novel nutrition-based biomarker that has been reported for predicting survival in various cancers. However, the relationship between CONUT score and prognosis of urological cancers remains unclear. Hence, we performed this meta-analysis to evaluate the prognostic significance of CONUT score for patients with urological cancers.

Condition being studied: Urological cancers, mostly containing renal cell carcinoma (RCC), prostate cancer (PC) and urothelial cancer (UC), are the major public health problem around the world. Despite the progress of the therapies and techniques for urological cancers including chemotherapy and molecular targeted therapy, the clinical prognosis of urological cancers remains not significantly increase in the past two decades, partly due to recurrence and metastasis. The current stage system is not enough to support the choice of treatment and the evaluation of prognosis of urological cancers. Therefore, it is critical to explore a new prognostic biomarker to guide the treatment of urological cancers.

INPLASY registration number: This protocol was registered with the International Platform of Registered Systematic Review and Meta-Analysis Protocols (INPLASY) on 23 December 2020 and was last updated on 23 December 2020 (registration number INPLASY2020120111).

\section{INTRODUCTION}

Review question / Objective: Controlling Nutritional Status (CONUT) score is a novel nutrition-based biomarker that has been reported for predicting survival in various cancers. However, the relationship between CONUT score and prognosis of urological cancers remains unclear. Hence, we performed this meta-analysis to evaluate the prognostic significance of CONUT score for patients with urological cancers. 
Condition being studied: Urological cancers, mostly containing renal cell carcinoma (RCC), prostate cancer (PC) and urothelial cancer (UC), are the major public health problem around the world. Despite the progress of the therapies and techniques for urological cancers including chemotherapy and molecular targeted therapy, the clinical prognosis of urological cancers remains not significantly increase in the past two decades, partly due to recurrence and metastasis. The current stage system is not enough to support the choice of treatment and the evaluation of prognosis of urological cancers. Therefore, it is critical to explore a new prognostic biomarker to guide the treatment of urological cancers.

\section{METHODS}

Participant or population: Patients were histopathologically diagnosed with urological cancers.

\section{Intervention: High CONUT score.}

Comparator: Patients with low CONUT score were controls.

Study designs to be included: Retrospective cohort study

Eligibility criteria: (1) patients were histopathologically diagnosed with urological cancers, including RCC, BC, PC UTUC; (2) reported the relationship between pretreatment CONUT score and overall survival (OS) or cancer-specific survival (CSS) or recurrence/disease/ progress-free survival (RFS/DFS/PFS); (3) provided hazard ratio (HR) and $95 \%$ confidence intervals $(\mathrm{Cl})$ directly.

Information sources: PubMed, Embase, the Cochrane Library and National Knowledge Infrastructure (CNKI) databases.

Main outcome(s): The pooled hazard ratios (HRs) with $95 \%$ confidence intervals (Cls) were calculated to evaluate the association of CONUT score with overall survival (OS).
Quality assessment / Risk of bias analysis: The quality of all primary studies were independently assessed in the light of the Newcastle-Ottawa Scale (NOS). Potential publication bias was evaluated by Begg's test with funnel plots.

Strategy of data synthesis: HR and $95 \% \mathrm{CI}$ were directly extracted from each publication to evaluate the importance of prognostic role of CONUT score for patients with urological cancers. To pool the overall $\mathrm{HR}$ with $95 \% \mathrm{Cl}$, the $\mathrm{HR}$ from the multivariate analysis was extracted from each study. The Cochran $Q$ and 12 statistical methods were applied to evaluate the heterogeneity among included studies. A fixed-effects model was used to calculate the pooled estimates in the absence of heterogeneity (120.10). Otherwise, a random-effects model was applied.

Subgroup analysis: According to cancer type, cancer stage, treatment methods, sample size and cut-off value, further subgroup analysis was also conducted.

Sensibility analysis: A sensitivity analysis was carried out to assess the influence of each individual study on the pooled results by sequentially excluding each study.

Country(ies) involved: China.

Keywords: CONUT score; urological cancers; meta-analysis; prognosis.

Contributions of each author:

Author 1 - Xinhao Niu.

Author 2 - Zhe Zhu.

Author 3 - Juan Bao. 